\title{
A case report of an eczematous facial and neck rash as a result of dupilumab
}

\author{
Selena R. Pasadyn', Urvashi Kaw², Daniel Knabel ${ }^{2,3}$
}

${ }^{1}$ Cleveland Clinic Lerner College of Medicine of Case Western Reserve University, USA, ${ }^{2}$ Department of Dermatology, Cleveland Clinic, USA, ${ }^{3}$ Department of Dermatopathology, Cleveland Clinic, USA

Corresponding author: Selena R. Pasadyn, E-mail: pasadys@ccf.org

\begin{abstract}
Dupilumab, an interleukin-4 receptor alpha antagonist, is an effective treatment for moderate-to-severe atopic dermatitis. Here we report the case of a 47-year-old male with an eczematous facial and neck rash of 4 weeks duration as a result of one year of dupilumab therapy. This patient presented with erythematous plaques along the nasolabial folds, with similar scattered papules on his cheeks and forehead, along with bilateral eye redness and edema. He had initial improvement on oral valcyclovir, but two weeks later presented with erythematous plaques involving the forehead, bilateral upper and lower eyelids, nose and cheeks, with accompanying yellow dry crusting. It was concluded that his initial rash was eczema herpeticum given improvement on valcyclovir. His second eruption was likely a dupilumab induced eczematous head and neck rash. The patient was placed on triamcinolone $0.1 \%$ ointment twice daily for 7-10 days and was instructed to stay off dupilumab and substantially improved.
\end{abstract}

Key words: Dupilumab; Eczema; Herpes; Adverse reaction

\section{INTRODUCTION}

Atopic dermatitis is an inflammatory skin disease presenting commonly on the hands and feet in adults [1]. It can cause significant dryness, pruritus, redness, swelling, scaly skin, crusting, and/or oozing. Atopic dermatitis follows a relapsing and remitting course, and patients can experience flares when triggers present in their environment, such as contact allergens or other irritants [2].

Common first line treatments for atopic dermatitis include topical steroids and calcineurin inhibitors [3]. However, when the condition becomes refractory, systemic agents can be employed. These include oral corticosteroids, cyclosporine, azathioprine, and mycophenolate $[2,4]$. Injectable dupilumab, an interleukin-4 (IL-4) receptor alpha antagonist, is also indicated for the treatment of adults with moderate-to-severe atopic dermatitis. It is typically prescribed for those who cannot achieve control topically, or may have contraindications to other systemic medications [2].

While dupilumab has shown significant effectiveness for atopic dermatitis, as well as improvements in patient quality of life, there are adverse effects [5]. The most common adverse reactions are injection-site reactions, conjunctivitis, blepharitis, keratitis, eye pruritus, oral herpes or other herpes simplex virus infections, and dry eyes $[5,6]$. However, only a few case reports have found eczematous facial and neck rashes as a result of the medication. Here we present the case of a 47-year-old male with a facial rash of 4 weeks duration as a result of dupilumab therapy.

\section{CASE REPORT}

One month ago, a 47-year-old male presented to the emergency department with a history of dry cough, sore throat, ear pain and bilateral conjunctivitis with eye pain, purulent discharge, blurry vision, and

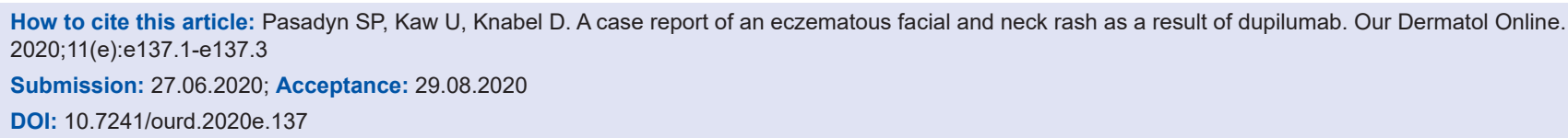


photosensitivity. Two days after these symptoms developed, he noticed a neck and facial rash that worsened despite taking diphenhydramine as needed. The patient denied any recent triggers for the rash, including changing detergent/soaps, new animals, or other changes to his daily routine. The patient went to the emergency department because of the rash and was prescribed methylprednisolone $4 \mathrm{mg}$ tablets in a dose pack and triamcinolone acetonide $0.5 \%$ twice daily.

Despite this treatment, the patient continued to worsen. He went to his primary care provider (PCP) two days later. The patient's PCP described the rash as raised, lightly erythematous papules covering his face. The patient was instructed to stop his current treatments and was placed on a prednisone $20 \mathrm{mg}$ tablet with a taper, hydroxyzine $\mathrm{HCl} 25 \mathrm{mg}$ as needed every 6 hours, and Systane artificial tears for symptomatic relief. However, none of these treatments improved his symptoms.

Three days later the patient presented to dermatology. At this visit, he was noted to have erythematous plaques on face along the nasolabial folds, with similar scattered papules on his cheeks and forehead. He also had bilateral eye redness and mild edema of his eyelid margins. The dermatologist prescribed him oral valcyclovir due to possible concern of dupilumab induced herpes simplex infection. After approximately five to seven days, the patient saw some improvement and the therapy caused the rash to dry up.

Three days later however, the patient returned to his PCP with continued cough symptoms. He was then started on ten days of doxycycline $100 \mathrm{mg}$ twice daily for suspected bronchitis. Throughout the ten days on the medication, the patient felt that his rash improved, but after discontinuing the doxycycline, the rash returned to his face and bilateral arms. At this point the patient returned to the emergency department.

On physical exam in the emergency department he was examined by dermatology and noted to have erythematous plaques involving the forehead, bilateral upper and lower eyelids, nose and cheeks. There was also yellowish dry crusting on the cheeks (Fig. 1). He also had scattered erythematous papules and plaques, some with scaling, seen on flexor left upper extremity. Labs revealed his CBC and CMP were within normal limits, only notable for low platelets with a count of

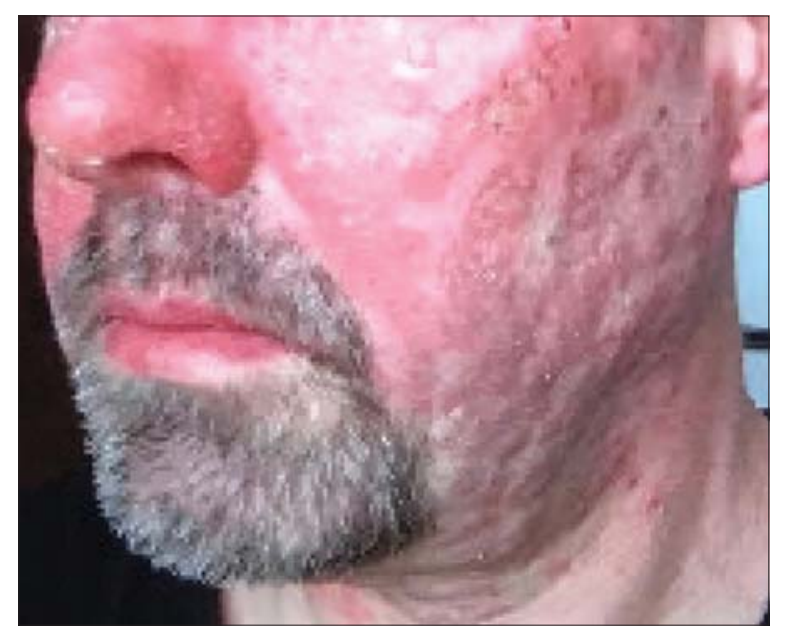

Figure 1: Erythematous plaques involving the forehead, bilateral upper and lower eyelids, nose and cheeks; yellowish dry crusting on the cheeks.

144,000. Liver function tests, amylase, lipase, hepatitis panel, HIV, erythrocyte sedimentation rate, and C3/C4 were all within normal limits. He has a slightly elevated C-reactive protein at 2.1 (normal $<0.9)$.

The patient's past medical history is notable for five years of chronic atopic dermatitis, or eczema, mostly confined to his hands and legs. He was started on dupilumab one year ago and received his last dose eight days before his first presentation to the emergency department. His other medical history includes a right upper extremity deep vein thrombosis (currently on apixaban), and alcohol (6-12 drinks per day) and nicotine abuse ( 1 pack per day for 20 years).

Overall, after this second emergency department visit, dermatology concluded that his initial rash was most likely eczema herpeticum induced by the dupilumab, as it responded to the oral valacyclovir. However, his second rash was more eczematous and likely a dupilumab induced eczematous head and neck rash. The patient was placed on triamcinolone $0.1 \%$ ointment twice daily on affected areas for 7-10 days and was instructed to stay off dupilumab. This resulted in significant improvement in the facial rash (Fig. 2).

\section{DISCUSSION}

This case report highlights the rare adverse cutaneous effect of dupilumab, an eczematous head and neck rash. While highly unusual, a few other case reports have reported similar findings. In one case, a 26-yearold female on dupilumab had fine scaling edematous 


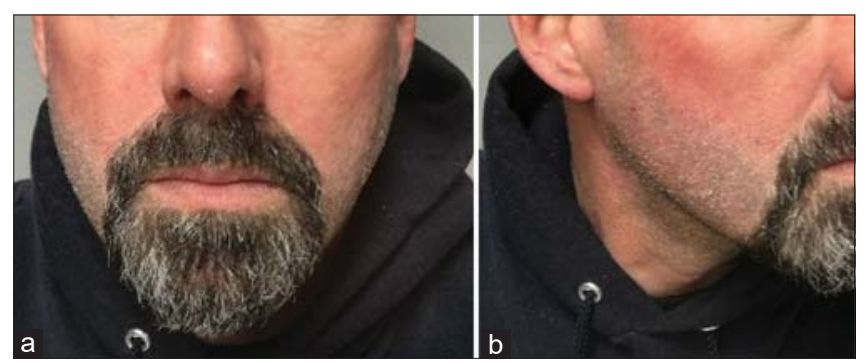

Figure 2: The patient experienced significant improvement in his facial rash after using triamcinolone $0.1 \%$ ointment twice daily for $7-10$ days and discontinuing the dupilumab.

pink red plaques on her face and left anterolateral neck six months into treatment. The patient was tried on topical treatment, oral prednisone, fluconazole, and doxycycline unsuccessfully, as she did not want to discontinue her dupilumab [7]. Another case report found that a 28-year-old female had experienced erythematous and slightly edematous patches over her cheeks and neck after 4 months on dupilumab [8]. She had relief with hydroxyzine and mometasone furoate cream twice daily. Beyond these cases, a larger scale study among 1,000 adult patients treated for atopic dermatitis with dupilumab found that $4.2 \%$ had head and neck dermatitis as a result. Seventy-five percent of those were an aggravation of already present dermatitis, and $25 \%$ were new cases [9].

Our patient also experienced an initial rash of eczema herpeticum possibly induced by the dupilumab. Eczema herpeticum is more common in those with atopic dermatitis than the general population. Interestingly though, in those with moderate-to-severe atopic dermatitis, it has been shown that dupilumabtreated patients have lower rates of clinically important herpes infections, such as eczema herpeticum and herpes zoster, than those treated with placebo [10].

In terms of a mechanism, dupilumab blocks IL-4, it therefore also modulates T-helper signaling. This imbalance could unmask a baseline allergic contact dermatitis [9]. Another proposed mechanism for this eczematous dupilumab reaction is that the IL-4 blockade could lead to an overgrowth of Malassezia fungus in sebaceous areas. It has been shown that there is a higher frequency of Malasseziaresponsive memory $\mathrm{CD} 4^{+} \mathrm{T}$ cells in patients with atopic dermatitis compared to controls [11].

Overall, it is important for providers to remember that in patients on dupilumab, an eczematous head and neck rash can result. Discontinuing dupilumab is the best approach to treating the problem, as the atopic dermatitis does not always remit easily while still on the drug. However, if dupilumab is vital to treating the underlying eczema, then a combination of tacrolimus, topical steroids, or antifungal agents may be useful.

\section{Consent}

The examination of the patient was conducted according to the principles of the Declaration of Helsinki.

The authors certify that they have obtained all appropriate patient consent forms, in which the patients gave their consent for images and other clinical information to be included in the journal. The patients understand that their names and initials will not be published and due effort will be made to conceal their identity, but that anonymity cannot be guaranteed.

\section{REFERENCES}

1. Nutten S. Atopic dermatitis: global epidemiology and risk factors. Ann Nutr Metab. 2015;66 Suppl 1:8-16.

2. D'Ippolito D, Pisano M. Dupilumab (Dupixent): an interleukin-4 receptor antagonist for atopic dermatitis. Pharm Ther. 2018;43:532-5.

3. Lyons JJ, Milner JD, Stone KD. Atopic dermatitis in children. Immunol Allergy Clin. 2015;35:161-83.

4. Buys LM. Treatment options for atopic dermatitis. Am Fam Physician. 2007;75:523-8.

5. Dupixent (dupilumab) prescribing information. Tarrytown, New York: Regeneron Pharmaceuticals; Mar, 2017.

6. Ou Z, Chen C, Chen A, Yang Y, Zhou W. Adverse events of Dupilumab in adults with moderate-to-severe atopic dermatitis: A meta-analysis. Int Immunopharmacol. 2018;54:303-10.

7. Dalia Y, Marchese Johnson S. Case report: first reported case of facial rash after dupilumab therapy. Pract Dermatol. 2018;Apr:25-6.

8. Albader SS, Alharbi AA, Alenezi RF, Alsaif FM. Dupilumab side effect in a patient with atopic dermatitis: a case report study. Biologics. 2019;13:79-82.

9. Soria A, Du-Thanh A, Seneschal J, Jachiet M, Staumont-Salle D, Barbarot S. Development or exacerbation of head and neck dermatitis in patients treated for atopic dermatitis with dupilumab. JAMA Dermatol. 2019;155:1312-5.

10. Eichenfield LF, Bieber T, Beck LA, Simpson EL, Thaci D, BruinWeller $\mathrm{M}$, et al. Infections in dupilumab clinical trials in atopic dermatitis: a comprehensive pooled analysis. Am J Clin Derm. 2019;20:443-56.

11. Sparber F, De Gregorio C, Steckholzer S, Ferreira FM, Dolowschiak T, Ruchti F, et al. The skin commensal yeast malassezia triggers a type 17 response that coordinates anti-fungal immunity and exacerbates skin inflammation. Cell Host Microbe. 2019;25:389-403.e6.

Copyright by Selena R. Pasadyn, et al. This is an open access article distributed under the terms of the Creative Commons Attribution License, which permits unrestricted use, distribution, and reproduction in any medium, provided the original author and source are credited.

Source of Support: Nil, Conflict of Interest: None declared. 